\title{
Effect of some nanoparticles on the stages biology of the southern cowpea beetle Callosobruchus maculatus (Fab.) (Coleoptera:Bruchidae)
}

\author{
Adnan Mosa Mohammed \\ Sarah Amer Aswd ${ }^{1}$ \\ Department of biology / College of Education For Pure Science \\ University of Mosul \\ adnanmosa29@yahoo.com \\ sarah1991amer@gmail.com
}

DOI: $\underline{10.33899 / \text { edusj.2019.162956 }}$

Received
$24 / 12 / 2018$

\section{Accepted}

29 / 01 / 2019

\begin{abstract}
Nano particle of Zinc oxide size $(5 \mathrm{~nm})$ and $(100 \mathrm{~nm})$ and silver nano Particle $(100 \mathrm{~nm})$ were used in This study at concentrations $(100,250,500,750$ and 1000) ppm to know their effect on some biological aspects of the southern cowpea beetle Callosobruchus maculatus (Fab.) through treating the food of these insects (chick pea seeds) by these nanoparticles.

The study proved that the above Particles had a significant effect on the parentage of eggs hatching as it reached $49.4 \%$ compared to $86.9 \%$ of control Also, the some treatment led to high at 1000 ppm concentration mortality percentage $98 \%$. Moreover, the treatment had an effect on the pupa stage and on the period of insect development, which was elongated significantly in the treatments of the zinc oxide size $(100 \mathrm{~nm})$. The maximum period was 40.60 days at the concentration of $1000 \mathrm{ppm}$ Which differed significantly from its comparative coefficient. The productivity of insect decreased significantly to $16.6 \%$ in zinc oxide nano Particle size $(5 \mathrm{~nm})$ clearly. This study showed that the treatments effected the vitality of the first-generation females as the percentage of egg hatching decreased to $50 \%$ more than that egg retention was observed on these females which reached to $50 \%$ at treatment of zinc oxide size (5 nanometer).
\end{abstract}

Keyword: Callosobruchus maculatus, zinc oxide nano Particle,silver nano Particle.

$$
1 \text { بحث مستل من رسالة الماجستير للباحث الثاني بأشراف الباحث الأول }
$$




\title{
Callosobruchus تأثير بعض المواد النانوية في حيوية أطوار خنفساء اللوبيا الجنوبية (Colob) maculatus (Fab.) (Coleoptera:Bruchidae)
}

\author{
سارة عامر أسود \\ عدنان موسى محمد \\ قسم علوم الحياة / كلية التربية للعلوم الصرفة

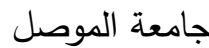

sarah1991amer@gmail.com

DOI: $\underline{10.33899 / \text { edusj.2019.162956 }}$

القبول

2019 / $01 / 29$

\section{adnanmosa29@yahoo.com}

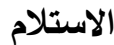

2018 / 12 / 24

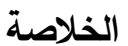

أستعملت في هذه الدراسة المواد النانوية أوكسيد الزنك النانوي حجم (5) و(100) نانوميتر ومادة

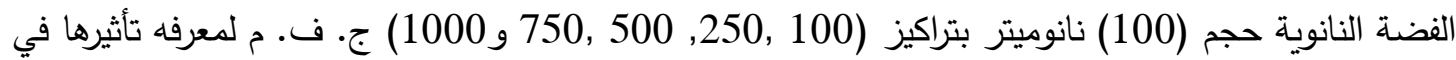
حيوية اطوار خنفساء اللوبيا الجنوبية Callosobrchus maculatus من خلال معاملة غذائها (بذور الحمص)

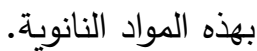

أثتبت الدراسة الحالية أن المواد النانوية المستعملة أثرت معنويا في حياتية الحشرة أذ سببت خفضاً معنوياً في نسبة فقس البيض الى 49.4\% بينما كانت نسبة فقس بيض المقارنة 86.9\% كما أدت إلى نسبة قتل عالية للطور اليرقي وصلت إلى 98\% في معاملة أوكسيد الزنك النانوي حجم (5) نانوميتر فضلاً عن ذلك لكانك

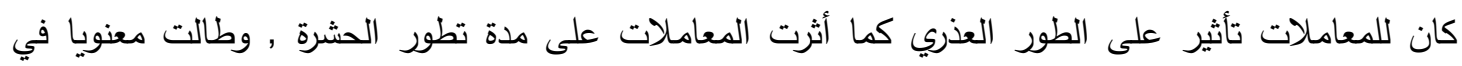

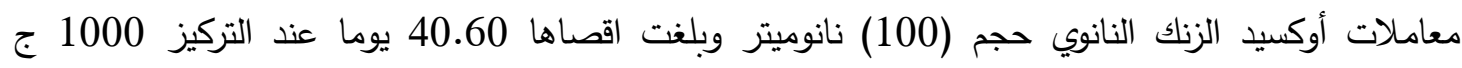

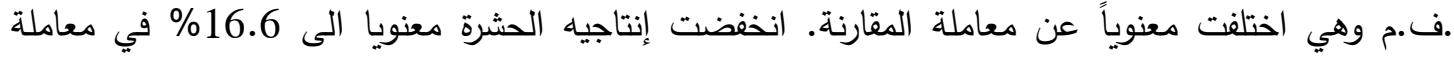
أوكسيد الزنك النانوي حجم (5) نانومتر أثبتت هذه الدراسة ان المعاملات اثرت على حيوية اناث الجيل الاول إذا فئا

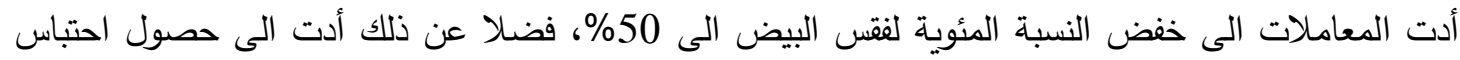
البيض لهذه الإناث ووصل الى حدود 50 \% في معاملة أوكسيد الزنك النانوي حجم (5) نانوميتر .

الكلمات المفتاحية: خنفساء اللوبيا الجنوبية, أوكسيد الزنك النانوي, الفضة النانوية. المقدمة

تعد المواد المخزونة بشكل عام الحبوب والبقوليات على وجه الخصوص ذات أهمية أذ تحرص جميع

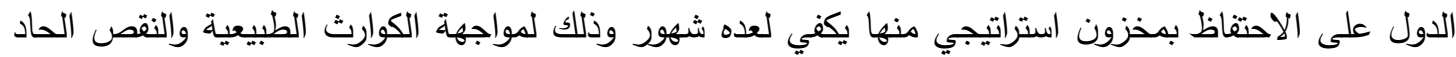
في الانتاج السنوي الغذائي وتتعرض هذه المواد المخزونة للتلف بفعل العديد من الأحياء كالحشرات والقوارض لفرون وبذلك تفسد وتفقد قيمتها الغذائية[1].

وهذه البقوليات عرضة للإصابة بالعديد من الآفات من أكثر أنواع الآفات التي تصيب الهحاصيل

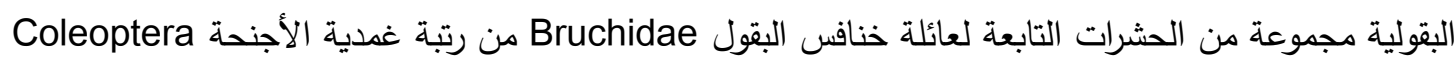
وذلك لقدرتها على التكاثر على البذور الجافة [2,3]. 
وتعد خنفساء اللوبيا الجنوبية Callosobruchus maculatus من اهم الحشرات التي تسبب أضرار

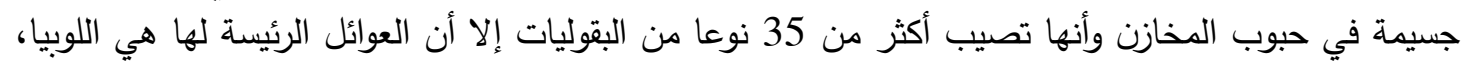

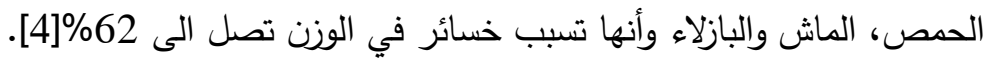

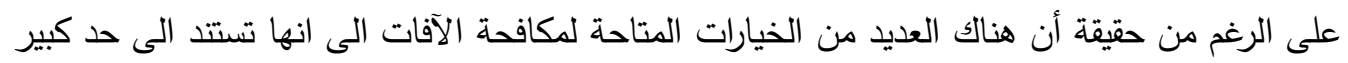

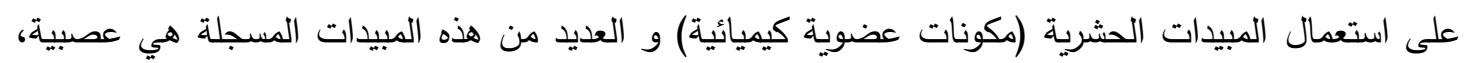

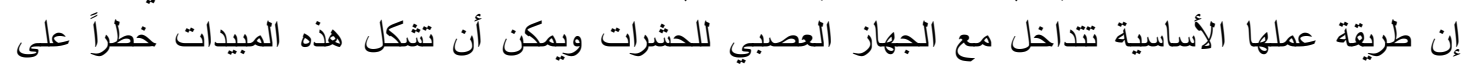
الثييات مثل هذه المبيدات تعمل على اخلال بالعمل ATP او على نمو الحشرات ومع تقديم الاساليب الحديثة

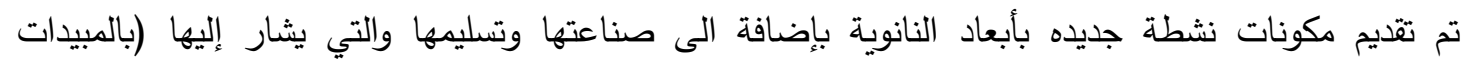

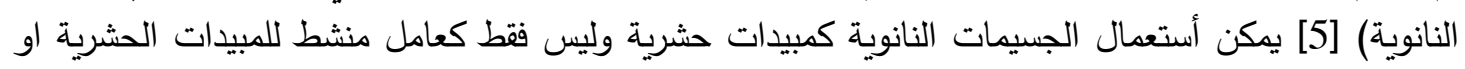

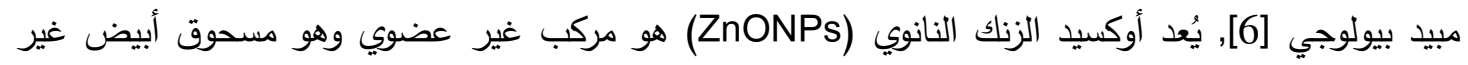

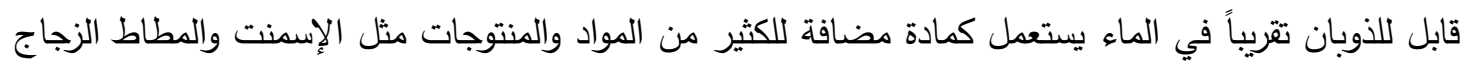

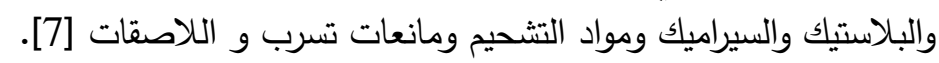
وكذلك تعتبر جسيمات الفضة النانوية (AgNPs) مهمة لأنها تستعمل في العديد من السلع الاستهلاكية العناد

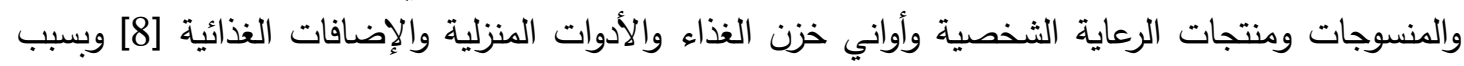

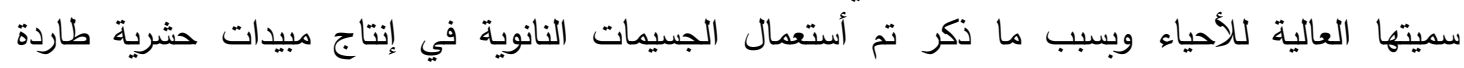

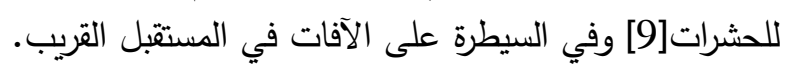

المواد وطرائق العمل

أجريت الدراسة في مختبرات كلية التربية للعلوم الصرفة قسم علوم الحياة تمت تربية الحشرة على بذور حمص في قنينة زجاجية كبيره بطول 20 سم و قطر 6سم وغطيت الفوهات بقماش الثاش الطبي لعده طبقات و

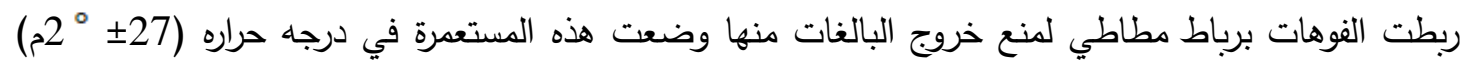
ورطوبة نسبية (60 × 5)\% الى حين خروج البالغات لغرض المعاملة و كانت المستعمرات تجدد باستمرار اذ يتم اخذ حشرات فتية منها باستمرار .

المواد النانوية المستعملة في الدراسة هي أوكسيد الزنك النانوي حجم (5) و(100) ونانية نانوميتر ومادة

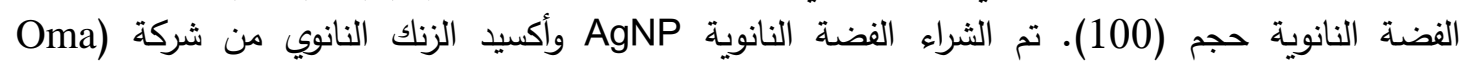
International Trading Authorized Partner of Sigma-Aldrich/ Germany) الثركة المصنعة فإن الفضة النانوية أقل من 100 نانوميتر تم قياس معدل حجم جسيماتها باستعمال صور المجهر الالكتروني الأنتالي او النافذ TEM. فقد استلمت على شكل مسحوق نانوي حجم الجسيمة فيه 100

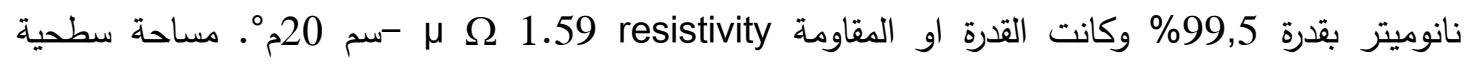

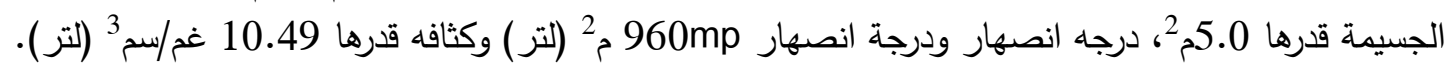

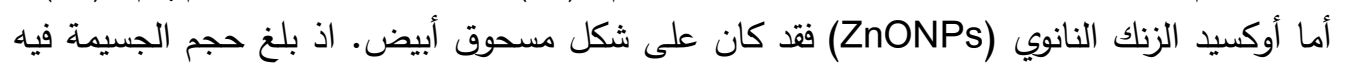

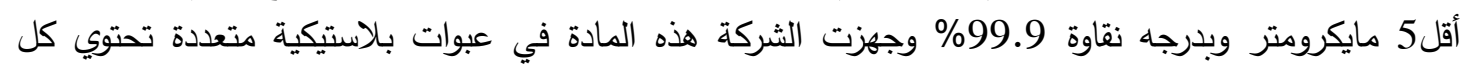

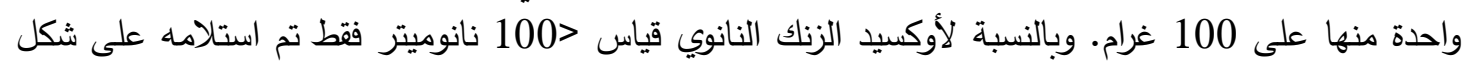

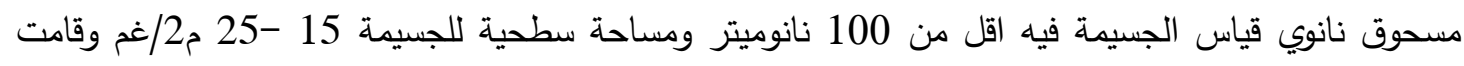
الشركة بتجهيز المادة على شكل عبوات متعددة تحتوي كل واحدة منها على 50 غم من المسحوق.

\section{تحضير المحاليل المستعملة في الاراسة}

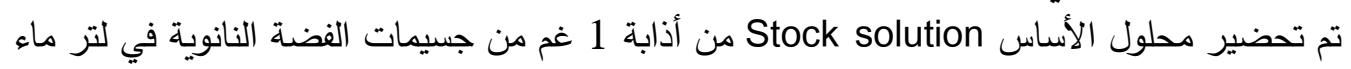

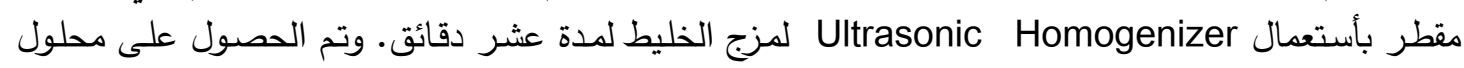
بتركيز 1000 جم. ف. 
اما أوكسيد الزنك النانوي فقد تم تحضير محلول الأساس Stock solution من أذابه 1 غم من المادة

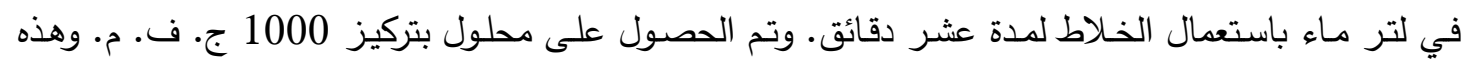

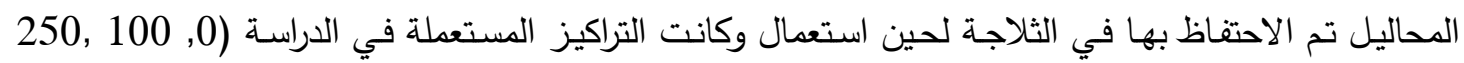
(1000, 750, 500, المقطر اما التراكيز الاخرى فقد حضرت من تخفيف محلول اساس بالماء المقطر .

الاراسة الحياتية: لمعرفة تأثير المادة النانوية التي تم تحضيرها مسبقا على حياتية خنفساء اللوبيا الجنوبية فقد تم نقع البذور السليمة للحمص في 4 مل من المادة ولكل التراكيز للمدة من 15 الى 20 دقيقة وهو وقت كاتِ كافِ

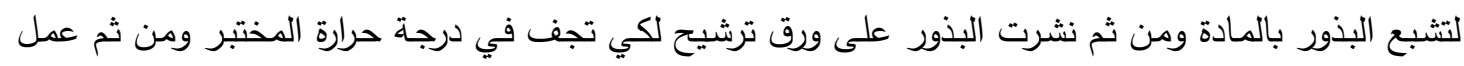

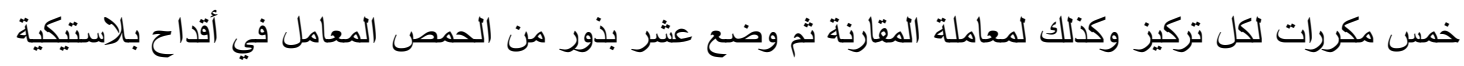

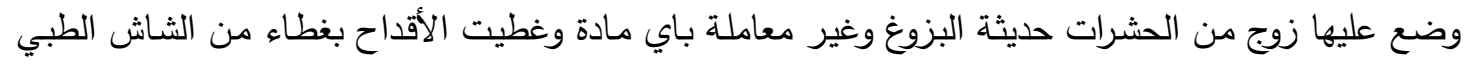

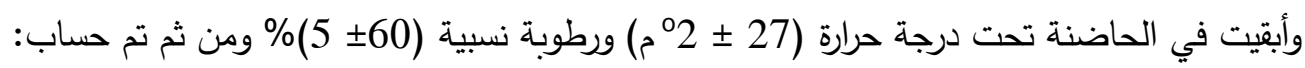

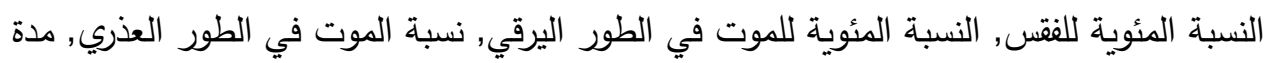

$$
\text { [10], التطور /يوم, الإنتاجية للإناث (الإنتاجية = ع عدد البيضن الموضواع الخارجة }
$$

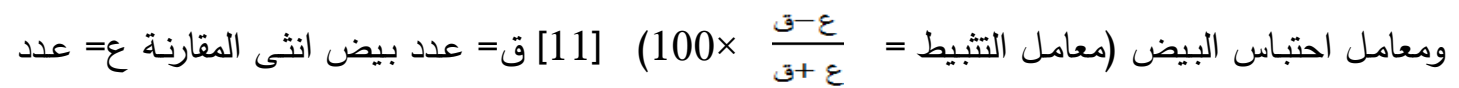

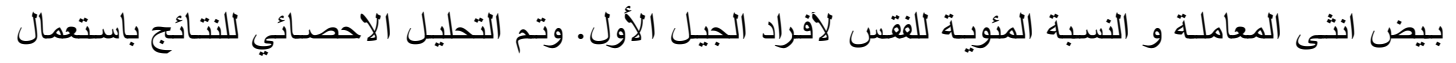

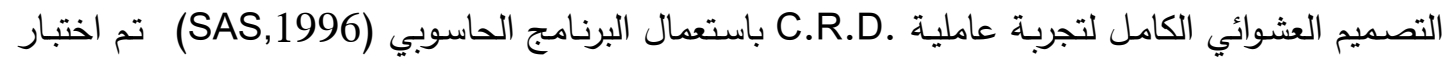
الفروقات بين المتوسطات باستعمال اختبار دنكن المتعدد المدى Duncan Multiple Rang تحت مستوى احتمال 50]\% [12].

\section{النتائج والمناقشة}

1- تأثير تراكيز مختلفة لثلاث مواد نانوية على النسبة المئوية لفقس البيض لخنفساء اللوبيا الجنوبية

\section{.Callosobruchus maculatus}

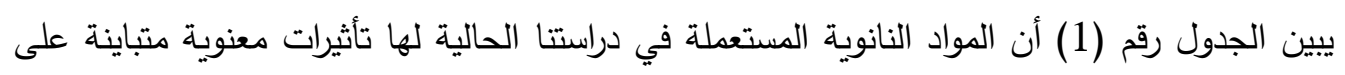
نسبة فقس البيض لخنفساء اللوبيا الجنوبية C. maculatus أذ بين الجدول أن المادة النانوية المستعملة قللت نسبة الفقس 76.39و76.92\% لمادتي أوكسيد الزنك النانوي 100 نانوميتر وأكسيد الزنك النانوي 5 نانوميتر التبن على التوالي وهي تختلف معنويا عن معاملة الفضة النانوية 100 نانوميتر والتي بلغت فيها نسبة الفتس .\%85.84

كما يبين الجدول رقم (1) أن التراكيز المستعملة في الدراسة لها تأثيرات متقاربة فكانت اقل نسبة 78

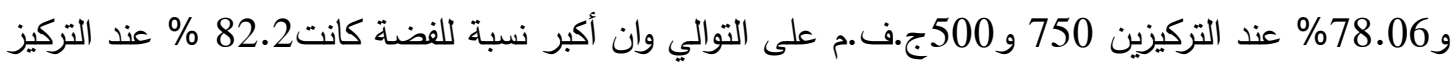
250ج.ف.م وهي تختلف معنويا عن بعضها البعض. وند كما يبين الجدول أن للتداخل ما بين نوع المادة النانوية وتراكيزها تأثيرات واضحة عضد على نسبة الفقس, اذ

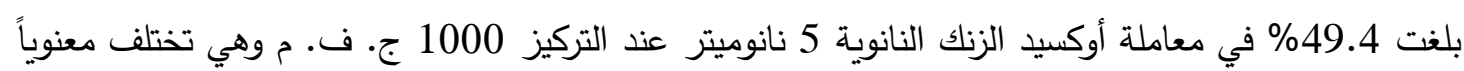
عن معاملة المقارنة و التي بلغت 86.94 \% في حين بلغت أقل نسبة فقس 65 \% في معاملة أوكسيد الزنك 
النانوي 100 نانوميتر عند التركيز 500ج .ف. م كان بينها و بين المعاملة السابقة فرقا معنويا راذ بلغت النسبة المئوية للفقس اكثر من 80\% في معاملة الفضة النانوية 100 نانوميتر و لجميع التراكيز المستعطلة و لم يكن لئن بينها فرقا معنويا و لا بينها و بين معاملة المقارنة.

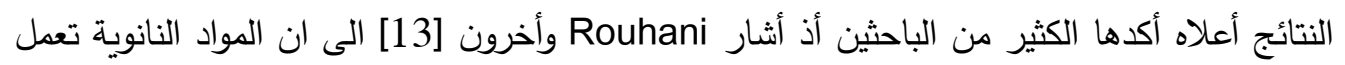
عمل المبيدات الحشرية في التأثير على حياتية حشرة خنفساء اللوبيا الجنوبية C. maculatus كما أشاروا

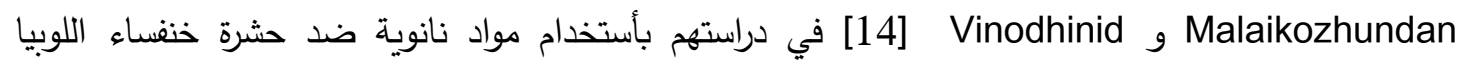

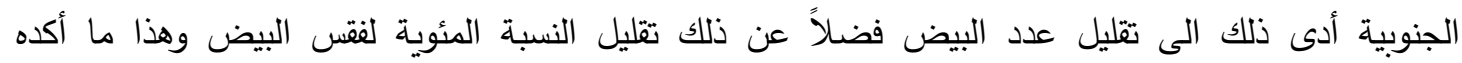
Athanassiou

الجدول رقم (1): تأثير تراكيز مختلفة لثلاث مواد نانوية على النسبة المئوية لفقس البيض لخنفساء اللوبيا الجنوبية

\section{Callosobruchus maculatus}

\begin{tabular}{|c|c|c|c|c|c|c|}
\hline \multirow[t]{2}{*}{ معدل تأثير المادة } & \multicolumn{5}{|c|}{ المئوية لفقس البيض } & \multirow[b]{2}{*}{ المادة } \\
\hline & 1000 & 750 & 500 & 250 & 100 & \\
\hline $\begin{array}{c}76.92 \\
b\end{array}$ & $\begin{array}{c}49.4 \\
\mathrm{~h}\end{array}$ & $\begin{array}{l}74 \\
\text { d-f }\end{array}$ & $\begin{array}{c}83.2 \\
\text { b-c }\end{array}$ & $\begin{array}{l}81.2 \\
\text { b-c }\end{array}$ & $\begin{array}{c}.886 \\
a-c\end{array}$ & أوكسيد الزنك النانوي حجم (5) نانوميتر \\
\hline $\begin{array}{c}76.36 \\
b\end{array}$ & $\begin{array}{c}95.6 \\
\mathrm{a}\end{array}$ & $\begin{array}{l}74 \\
d-f\end{array}$ & $\begin{array}{l}65 \\
f-g\end{array}$ & $\begin{array}{l}83.2 \\
b-e\end{array}$ & $\begin{array}{c}66.4 \\
f-g\end{array}$ & أوكسيد الزنك النانوي حجم (100) \\
\hline $\begin{array}{c}85.84 \\
\mathrm{a}\end{array}$ & $\begin{array}{r}87.8 \\
\text { a-b }\end{array}$ & $\begin{array}{r}86.4 \\
a-c\end{array}$ & $\begin{array}{r}88 \\
a-b\end{array}$ & $\begin{array}{r}82.2 \\
\text { b-e }\end{array}$ & $\begin{array}{r}84.8 \\
\text { b-d }\end{array}$ & الفضة النانوية حجم (100) نانوميتر \\
\hline \multicolumn{6}{|c|}{$\begin{array}{c}86.9 \\
\text { a-c }\end{array}$} & المقارنة \\
\hline & $\begin{array}{c}80.99 \\
\mathrm{a}\end{array}$ & $\begin{array}{c}78 \\
\mathrm{a}\end{array}$ & $\begin{array}{c}78.06 \\
\mathrm{a}\end{array}$ & $\begin{array}{c}82.2 \\
\text { A }\end{array}$ & $\begin{array}{c}79.33 \\
\mathrm{a}\end{array}$ & معدل تأثير التركيز \\
\hline
\end{tabular}

* الارقام التي تحمل أحرف مختلفة وفي الحقل نفسه تختلف عن بعضها احصائيا حسب اختبار دنكن المتعدد الددى وعند مستوى

2- تأثير تراكيز مختلفة لثلاث مواد نانوية على نسبة القتل في الطور اليرقي لخنفساء اللوبيا

\section{الجنوبية Callosobruchus maculatus.}

أثرت معاملة بذور الحمص بالمواد النانوية على حيوية الطور اليرقي فنلاحظ من الجدول رقم (2) هذه

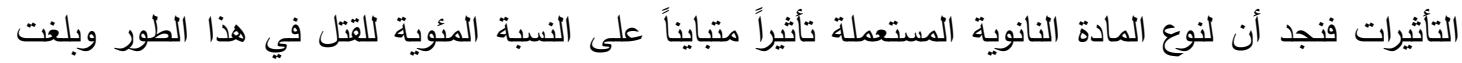

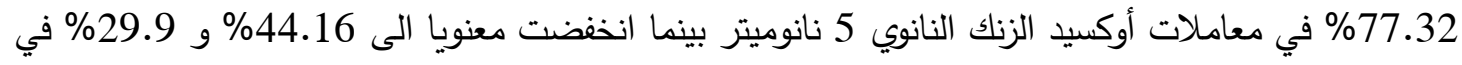

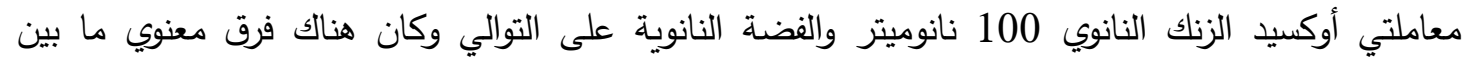
المعاملتين كلتيهما. فضلاً عن ذلك لاحظنا أيضا ان للتراكيز المختلفة للمواد النانوية تأثيرات متباينة فنجد من الجدول رقم (2) أن أقل نسبة للقتل بلغت 30.6\% عن التركيز 100 ج. ف. م وهي تختلف معنويا عن نسبة القتل في

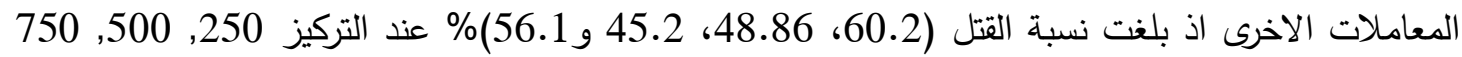
و1000 على التوالي وكان هنالك فرق ما بين المعاملات السابقة الذكر . 
كما يبين الجدول ان للتداخل ما بين المادة النانوي وتراكيزها تأثيراً متبايناً وبلغت أقل نسبة للقتل في

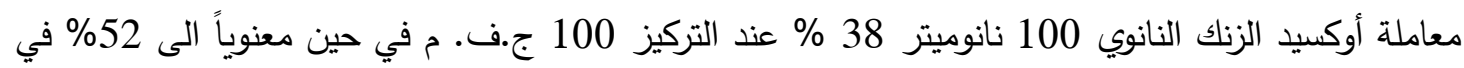
تركيز 500 ج. ف. م للمادة النانوية لنفسها.

ومن الجدول نلاحظ أن التراكيز المختلفة لمعاملات الفضة النانوية كان لها تأثير قليل في نسبة القتل الطور اليرقي لخنفساء اللوبيا الجنوبية و بلغت أقل نسبة 24.6\% تركيز 1000 ج. ف. م أختلفت النتائج

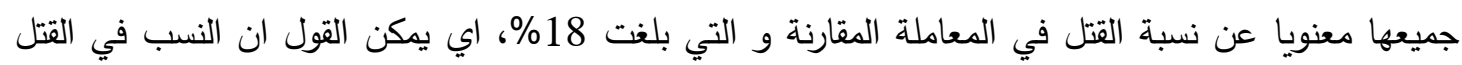

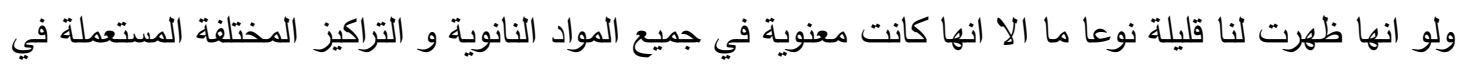

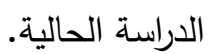

الجدول رقم (2): تأثير تراكيز مختلفة لثلاث مواد نانوية على نسبة الموت في الطور اليرقي لخنفساء اللوبيا الجنوبية

\section{Callosobruchus maculatus}

\begin{tabular}{|c|c|c|c|c|c|c|}
\hline \multirow[t]{2}{*}{ معدل تأثير المادة } & \multicolumn{5}{|c|}{ نسبة الموت في الطور اليرقي } & \multirow{2}{*}{ المادة } \\
\hline & 1000 & 750 & 500 & 250 & 100 & \\
\hline $\begin{array}{c}77.32 \\
\mathrm{a}\end{array}$ & $\begin{array}{c}98 \\
\mathrm{a}\end{array}$ & $\begin{array}{c}60.8 \\
c-d\end{array}$ & $\begin{array}{l}65 \\
\mathrm{c}\end{array}$ & $\begin{array}{c}77.8 \\
\mathrm{~b}\end{array}$ & $\begin{array}{c}85 \\
b\end{array}$ & أوكسيد الزنك النانوي حجم (5) نانوميتر \\
\hline $\begin{array}{c}44.16 \\
b\end{array}$ & $\begin{array}{c}45.8 \\
\text { e-f }\end{array}$ & $\begin{array}{l}45 \\
\text { e-f }\end{array}$ & $\begin{array}{l}52 \\
\text { d-e }\end{array}$ & $\begin{array}{l}40 \\
\mathrm{f}\end{array}$ & $\begin{array}{c}38 \\
\mathrm{f}-\mathrm{g} \\
\end{array}$ & أوكسيد الزنك النانوي حجم (100) نانوميتر \\
\hline $\begin{array}{c}29.6 \\
\mathrm{c}\end{array}$ & $\begin{array}{c}24.6 \\
\mathrm{~h}-\mathrm{i}\end{array}$ & $\begin{array}{c}30 \\
\text { g-h }\end{array}$ & $\begin{array}{c}29.6 \\
\text { g-h }\end{array}$ & $\begin{array}{c}63 \\
\mathrm{c} \\
\end{array}$ & $\begin{array}{l}30 \\
\text { g-h }\end{array}$ & الفضة النانوية حجم (100) نانوميتر \\
\hline \multicolumn{6}{|c|}{$\begin{array}{c}18 \\
\mathrm{j}\end{array}$} & 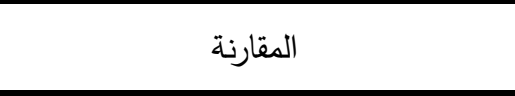 \\
\hline & $\begin{array}{c}65.1 \\
b\end{array}$ & $\begin{array}{c}45.2 \\
\mathrm{~d}\end{array}$ & $\begin{array}{c}48.86 \\
\mathrm{c}\end{array}$ & $\begin{array}{c}60.2 \\
a\end{array}$ & $\begin{array}{c}38.6 \\
\mathrm{e}\end{array}$ & معدل تأثير التركيز \\
\hline
\end{tabular}

* الارقام التي تحمل أحرف مختلفة وفي الحقل نفسه تختلف عن بعضها احصائيا حسب اختبار دنكن المتعدد المدى وعند مستوى احت احتمال5\%.

3- تأثير تراكيز مختلفة لثلاث مواد نانوية على معدل نسبة الموت في الطور العذري لخنفساء اللوبيا الجنوبية

\section{. Callosobruchus maculatus}

يبين الجدول رقم (3) أن المواد النانوية المستعملة في الدراسة الحالية أثرت على النسبة المئوية للموت

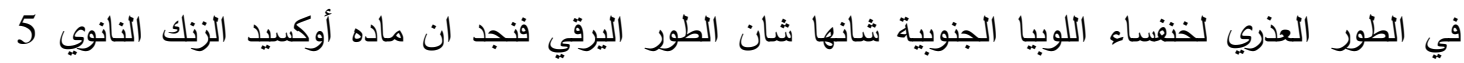
نانوميتر زادت نسبة القتل في الطور العذري معنويا الى 15.24\% وهي تختلف معنويا عن معاملتي المادتين

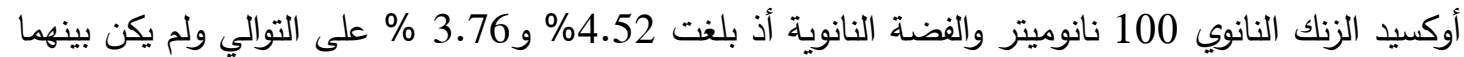

كما يبين الجدول (3) ان التراكيز المستعملة في الدراسة هي الاخرى لها تأثيرات متباينة على نسبة

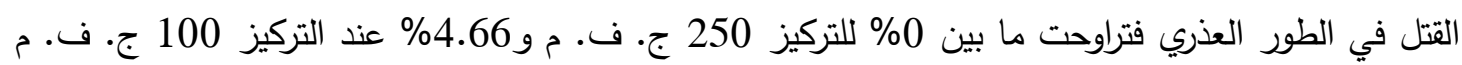
فضلا عن ذلك بلغت اعلى نسبة للقتل 21.93\% عند التركيز 500 ج. ف. ف. م. م.

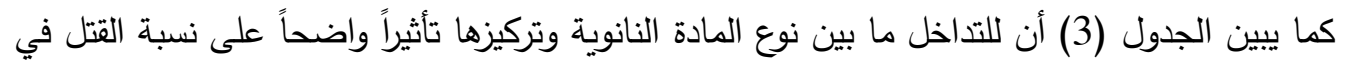
الطور العذري أذ بلغت أكبر نسبة 60\% في معاملة أوكسيد الزنك النانوي 5 نانوميتر عند التركيز 500ج.ف.م... 
أما في معاملة أوكسيد الزنك النانوي 100 نانوميتر نجد اعلى نسبة 10\% عند التركيز 1000 ج. ف.

م ولمعاملة الفضة النانوية اعلى نسبة للموت كانت 15\% في معاملة التركيز 1000 ج. ف. ف م واختلفت المعاملات اعلاه جميعا معنويا عن معاملة المقارنة ولم يحدث قتل في الطور العذري لكثير من المعاملات شانها شان معاملة المقارنة كما هو واضح من النتائج التي حصلنا عليها والموضحة في الجدول (3) وهذه فئه النتائج تثابهت مع دراسة Sahyaraj واخرون [17] إن المادة النانوية التي استعملناها في الدراسة أثرت على الطور العذري كما أثرت على الطور اليرقي الا انها بتركيز اقل كما تطابقت نتائجنا هنا الى حد معين مع نتائج Malaikozhundan بالمواد النانوية (أوكسيد الزنك النانوي).

وهذا ما اكده Rouhani واخرون [15] بتجربتهم على حشرة المن Aphis nerii من أن المواد النانوية

(الفضة النانوية و الزنك النانوي) تؤدي الى نسبة قتل 100\% في هذه الحشرة الحثة وهذا أيضا وضحه البندارني

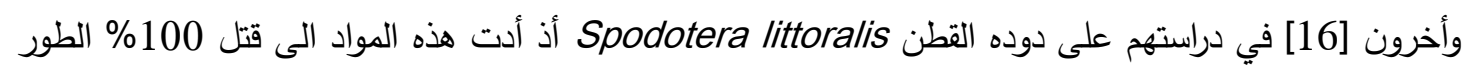
اليرقي.

جدول رقم (3): تأثير تراكيز مختلفة لثلاث مواد نانوية على معدل نسبة الموت في الطور العذري لخنفساء اللوبيا

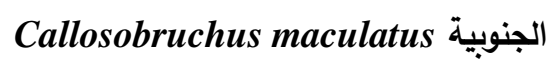

\begin{tabular}{|c|c|c|c|c|c|c|}
\hline \multirow{2}{*}{ المعادة } & \multicolumn{5}{|c|}{ معدل نسبة الموت في الطور العذري } & \multirow{2}{*}{ المادة } \\
\hline & 1000 & 750 & 500 & 250 & 100 & \\
\hline $\begin{array}{c}15.24 \\
\mathrm{a}\end{array}$ & $\begin{array}{l}4 \\
\mathrm{~b}\end{array}$ & $\begin{array}{l}10 \\
\mathrm{~b}\end{array}$ & $\begin{array}{c}60 \\
a\end{array}$ & $\begin{array}{l}0 \\
\mathrm{~b}\end{array}$ & $\begin{array}{c}2.2 \\
\mathrm{~b}\end{array}$ & أوكسيد الزنك النانوي حجم (5) نانوميتر \\
\hline $\begin{array}{c}4.52 \\
b\end{array}$ & $\begin{array}{c}10 \\
b\end{array}$ & $\begin{array}{c}2.2 \\
\mathrm{~b}\end{array}$ & $\begin{array}{l}2 \\
b\end{array}$ & $\begin{array}{l}0 \\
\mathrm{~B}\end{array}$ & $\begin{array}{c}8.4 \\
\mathrm{~b}\end{array}$ & أوكسيد الزنك النانوي حجم (100) \\
\hline $\begin{array}{c}3.76 \\
b\end{array}$ & $\begin{array}{c}15 \\
\mathrm{~b}\end{array}$ & $\begin{array}{l}0 \\
\mathrm{~b}\end{array}$ & $\begin{array}{c}3.8 \\
\mathrm{~b}\end{array}$ & $\begin{array}{l}0 \\
\mathrm{~B}\end{array}$ & $\begin{array}{l}0 \\
\mathrm{~b}\end{array}$ & الفضة النانوية حجم (100) نانوميتر \\
\hline \multicolumn{6}{|c|}{$\begin{array}{l}0 \\
b\end{array}$} & المقارنة \\
\hline & $\begin{array}{c}4.66 \\
b\end{array}$ & $\begin{array}{c}4.06 \\
b\end{array}$ & $\begin{array}{c}21.93 \\
\mathrm{a}\end{array}$ & $\begin{array}{l}0 \\
\mathrm{c}\end{array}$ & $\begin{array}{c}3.53 \\
b\end{array}$ & معدل تأثير التركيز \\
\hline
\end{tabular}

* الارقام التي تحمل أحرف مختلفة في الحقل نفسة تختلف عن بعضها احصائيا حسب اختبار دنكن المتعدد المدى وعند مستوى احتمال 5\%.

4- تأثير تراكيز مختلفة لثلاث مواد نانوية على مدة التطور/ يوم لخنفساء اللوبيا الجنوبية Callosobruchus maculatus

يبين الجدول رقم (4) أن المواد النانوية المستعملة في دراسة الحالية كان لها تأثيرات متباينة على مده تطور خنفساء اللوبيا الجنوبية من الجدول نجد أن المادة النانوية المستعملة أطالت هذه المدة معنويا فكانت 36.06 يوم في معاملة أوكسيد الزنك النانوي 100 نانوميتر وهي تختلف معنويا عن معاملتي أوكسيد الزنك الكادئ النانوي 5 نانوميتر والفضة النانوية أذ بلغت مدة التطور 29.92 يوماً 30.6 يوماً على التوالي ولم يكن بين المعاملتين فرقا معنويا. 
كما يبين الجدول رقم (4) أن للتراكيز المستعملة في الدراسة تأثيراً طفيفاً على مدة التطور فكانت أقل

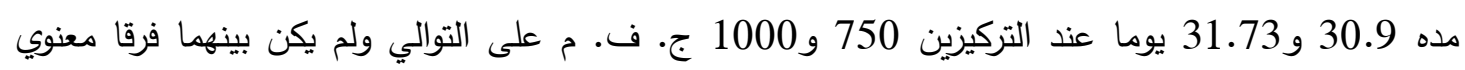

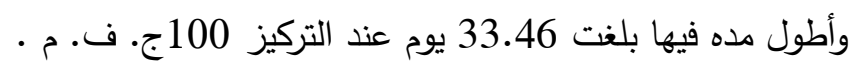
كما يبين الجدول أن للتداخل ما بين نوع المادة النانوية وتركيزها تأثيراً واضحاً على مدة التطور بلغت

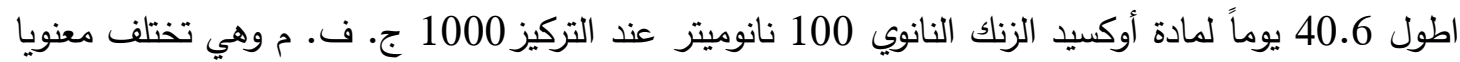

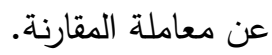

اما في المعاملة أوكسيد الزنك النانوي 5 نانوميتر فبلغت أطول مدة للتطور 35.4 يوما عند التركيز

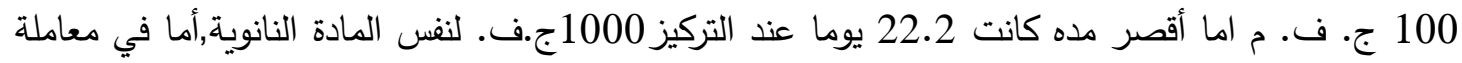
الفضة النانوية فكانت اطول مده 32.2 يوم عند التركيز 750ج.ف.م وأقل مده كانت 30 و 30.4 يوما عند التركيز 100 و1000ج.ف.م على التوالي. مما تقدم نجد أن مده التطور تأثرت بالتداخلات ما بين المادة النانوية وتراكيزها المختلفة في أغلب

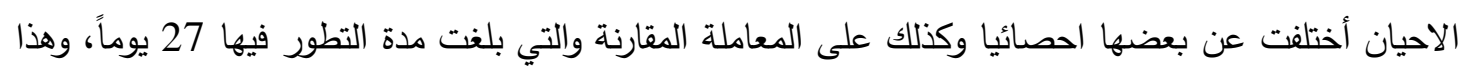
ما اكده Sahyaraj واخرون [17] ان المعاملات بالمواد النانوية أثرت معنويا على حياتية الحشرة Pericallia

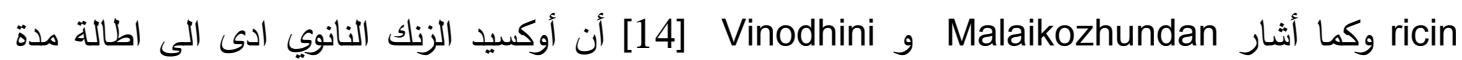
تطور خنفساء اللوبيا الجنوبية C maculatus . .

الجدول رقم(4): تأثير تراكيز مختلفة لثلاث مواد نانوية على مدة التطور/ يوم لخنفساء اللوبيا الجنوبية

Callosobruchus maculatus

\begin{tabular}{|c|c|c|c|c|c|c|}
\hline \multirow{2}{*}{ معدل } & \multicolumn{5}{|c|}{ مدة التطور ا يوم } & \multirow{2}{*}{ المادة } \\
\hline & 1000 & 750 & 500 & 250 & 100 & \\
\hline $\begin{array}{c}29.92 \\
\text { b }\end{array}$ & $\begin{array}{c}22.2 \\
\mathrm{e}\end{array}$ & $\begin{array}{c}28.6 \\
\text { c-e }\end{array}$ & $\begin{array}{l}31.8 \\
b-d\end{array}$ & $\begin{array}{l}31.6 \\
b-d\end{array}$ & $\begin{array}{c}35.4 \\
a-c\end{array}$ & أوكسيد الزنك النانوي حجم (5) نانوميتر \\
\hline $\begin{array}{c}36.06 \\
\mathrm{a}\end{array}$ & $\begin{array}{c}40.60 \\
\mathrm{a}\end{array}$ & $\begin{array}{c}34.4 \\
a-d\end{array}$ & $\begin{array}{c}37.40 \\
a-b\end{array}$ & $\begin{array}{c}33.8 \\
a-d\end{array}$ & $\begin{array}{c}34.6 \\
a-d\end{array}$ & أوكسيد الزنك النانوي حجم (100) \\
\hline $\begin{array}{c}30.6 \\
b\end{array}$ & $\begin{array}{c}30 \\
b-d\end{array}$ & $\begin{array}{c}32.2 \\
b-d\end{array}$ & $\begin{array}{c}29.2 \\
\text { c-e }\end{array}$ & $\begin{array}{c}31.6 \\
b-d\end{array}$ & $\begin{array}{c}30.4 \\
b-d\end{array}$ & الفضة النانوية حجم (100) نانوميتر \\
\hline \multicolumn{6}{|c|}{$\begin{array}{l}27 \\
d-e\end{array}$} & 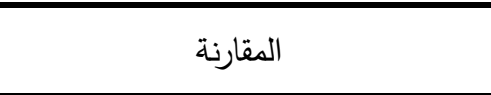 \\
\hline & $\begin{array}{c}30.9 \\
\mathrm{a}\end{array}$ & $\begin{array}{c}31.73 \\
\mathrm{a}\end{array}$ & $\begin{array}{c}32.66 \\
\mathrm{a}\end{array}$ & $\begin{array}{c}32.33 \\
\mathrm{a}\end{array}$ & $\begin{array}{c}33.46 \\
\mathrm{a}\end{array}$ & معدل تأثير التركيز \\
\hline
\end{tabular}

* الارقام التي تحمل أحرف مختلفة في الحقل نفسه تختلف عن بعضها احصائيا حسب اختبار دنكن المتعدد المدى وعند مستوى احتمال5\%.

5- تأثير تراكيز مختلفة لثلاث مواد نانوية على إنتاجية الحشرة لخنفساء اللوبيا الجنوبية

\section{Callosobruchus maculatus}

يعول الكثير من الباحثين على إنتاجية الحشرات لمعرفة كفاءة المواد المستعملة في مكافحة الحشرة فالمادة التي تقلل الإنتاجية هي التي تعتبر مفيدة في الحد من الكثافة العددية لتلك الافة وممكن الاستفادة منها في أدارة الافة. 
في دراستتا الحالية ومن الجدول (5) نجد أن الإنتاجية لخنفساء اللوبيا الجنوبية قد تأثرت بالمواد النانوية

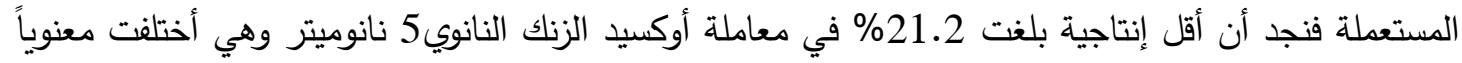

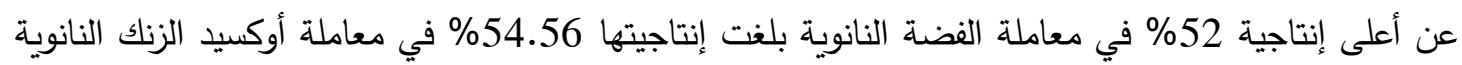

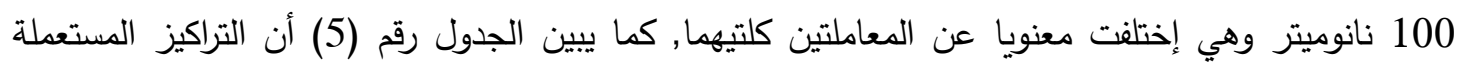

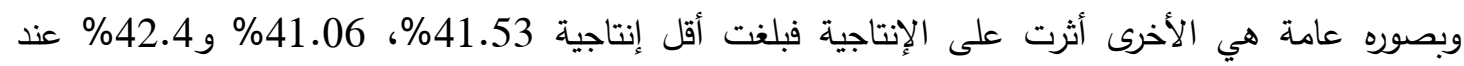

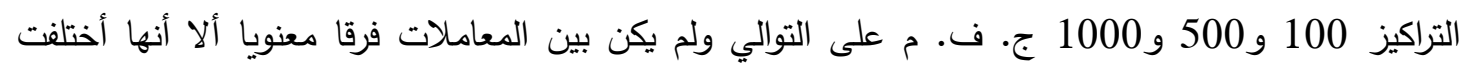

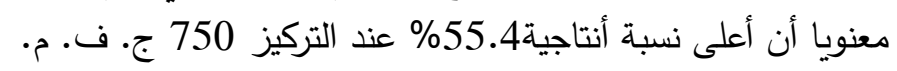

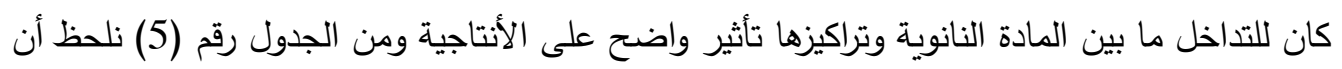

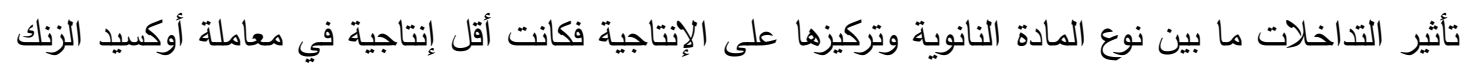

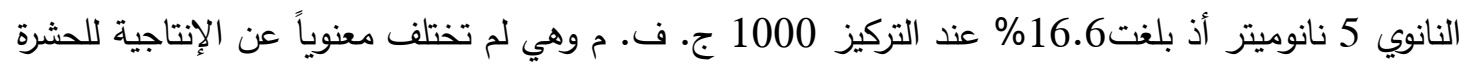

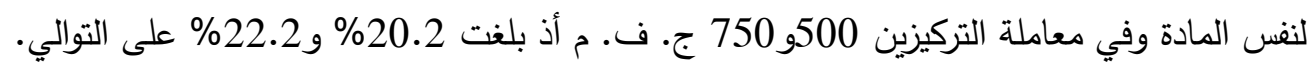

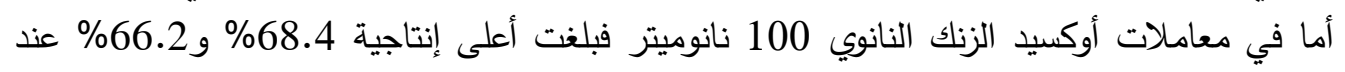

التركيز 250 و750ج.ف.م على التوالي ولم يكن بين هاتين المعاملتين فرق معنويٌ.

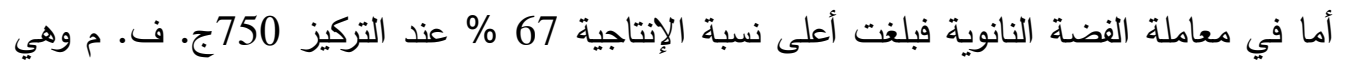

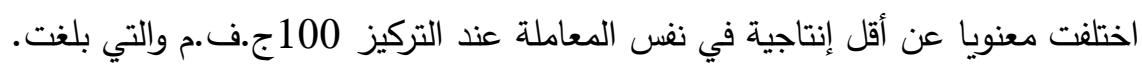

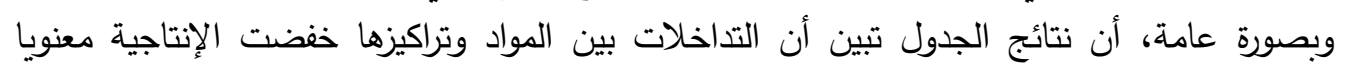
مقارنة مع إنتاجية المقارنة والتي بلغت 95\% وندة وهي تختلف معنوياً عن جميع المعاملات السابقة.

الجدول رقم(5). تأثير تراكيز مختلفة لثلاث مواد نانوية على إنتاجية حشرة خنفساء اللوبيا الجنوبية

\section{Callosobruchus maculatus}

\begin{tabular}{|c|c|c|c|c|c|c|}
\hline \multirow{2}{*}{ معدل تأثير المادة } & \multicolumn{5}{|c|}{ إنتاجية الحشرة } & \multirow{2}{*}{ المادة } \\
\hline & 1000 & 750 & 500 & 250 & 100 & \\
\hline $\begin{array}{c}21.2 \\
\mathrm{c}\end{array}$ & $\begin{array}{c}16.6 \\
\mathrm{~g}\end{array}$ & $\begin{array}{c}32.6 \\
\mathrm{f}\end{array}$ & $\begin{array}{c}22.2 \\
\mathrm{~g}\end{array}$ & $\begin{array}{c}20.2 \\
\mathrm{~g}\end{array}$ & $\begin{array}{c}37 \\
\mathrm{f}\end{array}$ & أوكسيد الزنكك النانوي حجم (5) نانوميتر \\
\hline $\begin{array}{c}54.56 \\
\mathrm{~b}\end{array}$ & $\begin{array}{c}57.8 \\
\text { b-d }\end{array}$ & $\begin{array}{c}66.2 \\
\mathrm{~b}\end{array}$ & $\begin{array}{c}41.4 \\
\text { e-f }\end{array}$ & $\begin{array}{c}68.4 \\
b\end{array}$ & $\begin{array}{l}39 \\
e-f\end{array}$ & أوكسيد الزنك النانوي حجم (100) نانوميتر \\
\hline $\begin{array}{c}58 \\
\mathrm{a}\end{array}$ & $\begin{array}{c}52.8 \\
\mathrm{c}-\mathrm{d}\end{array}$ & $\begin{array}{c}67 \\
\mathrm{~b}\end{array}$ & $\begin{array}{c}59.6 \\
\mathrm{~b}-\mathrm{c}\end{array}$ & $\begin{array}{c}61.6 \\
b-c\end{array}$ & $\begin{array}{c}48.6 \\
\mathrm{~d}-\mathrm{e}\end{array}$ & الفضة النانوية حجم (100) نانوميتر \\
\hline \multicolumn{6}{|c|}{$\begin{array}{c}95 \\
a\end{array}$} & المقارنة \\
\hline & $\begin{array}{c}42.4 \\
\mathrm{c}\end{array}$ & $\begin{array}{c}55.4 \\
\mathrm{a}\end{array}$ & $\begin{array}{c}41.06 \\
\mathrm{c}\end{array}$ & $\begin{array}{c}50 \\
\mathrm{~b}\end{array}$ & $\begin{array}{c}41.53 \\
\mathrm{c}\end{array}$ & معدل تأثير التركيز \\
\hline
\end{tabular}

* الارقام التي تحمل أحرف مختلفة في الحقل نفسه تختلف عن بعضها احصائيا حسب اختبار دنكن المتعدد المدى وعند مستوى

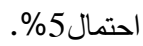

6- تأثير تراكيز مختلفة لثلاث مواد نانوية على معدل احتباس البيض لخنفساء اللوبيا الجنوبية Callosobruchus maculatus

إن هذه التجربة الغاية منها معرفة إمكانية انتقال تأثير معاملة الحشرات بالمواد النانوية الى الأفراد التي

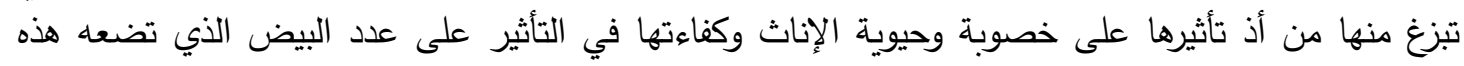


الإناث. الجدول رقم (6) بين أن للمعاملات تأثيرا واضحاً في ذلك نجد أن أعلى نسبة لاحتباس البيض بلغت

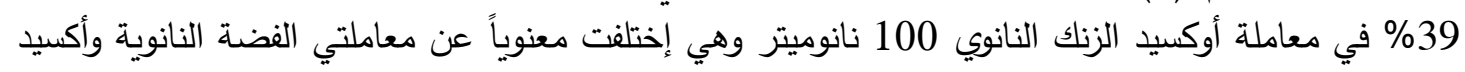

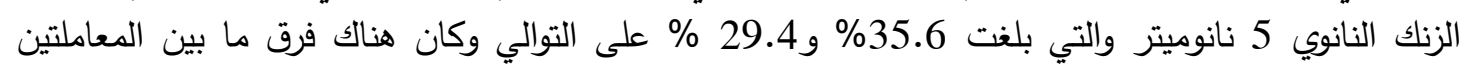
كلتيهما.

كما يبين الجدول رقم (6) أن التراكيز المستعملة في هذه الدراسة هي الأخرى أثرت على نسبة احتباس البيض لأفراد الجيل الاول من خنفساء اللوبيا الجنوبية اذ بلغت أعلى نسبة ف. م, في حين انخفضت الإنتاجية معنويا الى 29.3\% في معامل التركيز 100 ج. ف. م وهذه الأخيرة لم تختلف معنويا عن الإنتاجية في التراكيز الأخرة المستعملة في هذه الدراسة.

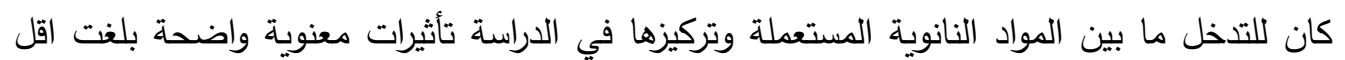

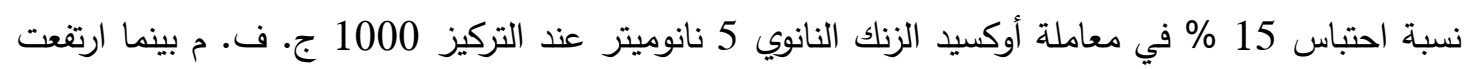

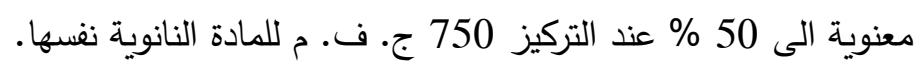
بلغت اعلى نسبة الاحتباس 48\% عند التركيز 750 ج. ف. م في معاملة أوكسيد الزنك النانوي 100 نانوميتر في حين انخفضت معنويا الى 21\% عند التركيز 100 ج. ف. م للمادة النانوية نغسها. كما يبين

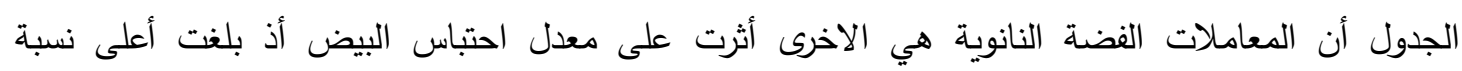
للاحتباس 47.3\% في التركيز 750ج.ف.م في حين أنخفضت الى 29.3، التراكيز 500,250,100, 1000 ج. ف. م على التوالي و لم يكن هناك فرق معنوي بين هذه المعاملات واختلفت

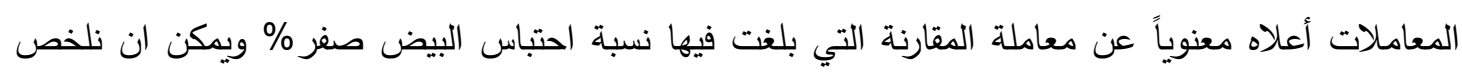

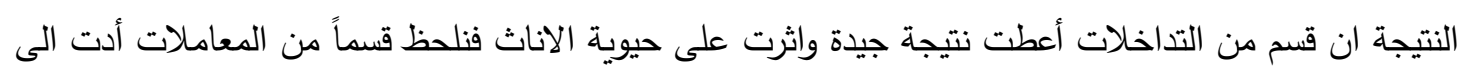
احتباس البيض بنسبة قاربت 50\% وهي نتيجة جيدة يمكن الاستفادة منها في مكافحة هذه الحشرة، اي ان

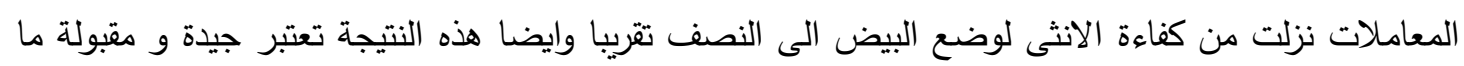

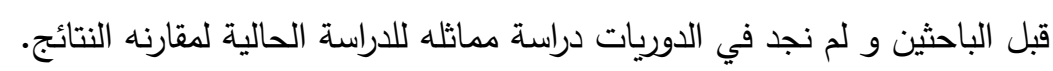

الجدول رقم (6): تأثير تراكيز مختلفة لثلاث مواد نانوية على معدل احتباس البيض لخنفساء اللوبيا الجنوبية

Callosobruchus maculatus

\begin{tabular}{|c|c|c|c|c|c|c|}
\hline \multirow{2}{*}{ معدل تأثير المادة } & \multicolumn{5}{|c|}{ معدل احتباس البيض } & \multirow{2}{*}{ المادة } \\
\hline & 1000 & 750 & 500 & 250 & 100 & \\
\hline $\begin{array}{c}29.4 \\
\text { c }\end{array}$ & $\begin{array}{c}50 \\
\mathrm{a}\end{array}$ & $\begin{array}{c}15 \\
\mathrm{f}\end{array}$ & $\begin{array}{c}30 \\
\mathrm{~d}\end{array}$ & $\begin{array}{c}23 \\
\mathrm{e}\end{array}$ & $\begin{array}{c}29 \\
\mathrm{~d}\end{array}$ & أوكسيد الزنك النانوي حجم (5) نانوميتر \\
\hline $\begin{array}{c}39 \\
\mathrm{a}\end{array}$ & $\begin{array}{l}48 \\
a-b\end{array}$ & $\begin{array}{c}39 \\
\mathrm{c}\end{array}$ & $\begin{array}{l}45 \\
a-c\end{array}$ & $\begin{array}{c}42 \\
b-c\end{array}$ & $\begin{array}{c}21 \\
\mathrm{e}\end{array}$ & أوكسيد الزنك النانوي حجم (100) \\
\hline $\begin{array}{c}35.6 \\
\mathrm{~b}\end{array}$ & $\begin{array}{l}47 \\
a-b\end{array}$ & $\begin{array}{l}44 \\
a-c\end{array}$ & $\begin{array}{l}20 \\
\text { e-f }\end{array}$ & $\begin{array}{c}29 \\
\mathrm{~d}\end{array}$ & $\begin{array}{c}38 \\
\mathrm{c}\end{array}$ & الفضة النانوية حجم (100) نانوميتر \\
\hline \multicolumn{6}{|c|}{$\begin{array}{l}0 \\
\mathrm{~g}\end{array}$} & المقارنة \\
\hline & $\begin{array}{c}48.33 \\
\mathrm{a}\end{array}$ & $\begin{array}{c}32.66 \\
\mathrm{~b}\end{array}$ & $\begin{array}{c}31.6 \\
b-c\end{array}$ & $\begin{array}{c}31.3 \\
b-c\end{array}$ & $\begin{array}{c}29.3 \\
\text { c }\end{array}$ & معدل تأثير التركيز \\
\hline
\end{tabular}

* الارقام التي تحمل أحرف مختلفة في الحقل نفسه تختلف عن بعضها احصائيا حسب اختبار دنكن المتعدد المدى وعند مستوى 
7- تأثير تراكيز مختلفة لثلاث مواد نانوية على نسبة الفقس البيض للجيل الاول لخنفساء اللوبيا الجنوبية

Callosobruchus maculatus

أجريت دراسة لمعرفة أمكانية انتقال تأثير المواد النانوية على نسبة الفقس لبيض الجيل الاول فنجد من الجدول رقم (7) أن أعلى نسبة فقس 84.34\% في معاملة أوكسيد الزنك النانوي 5 نانوميتر وانخفضت فئست معنويا الى 76.32\% و 71.3\% في معاملتي أوكسيد الزنك النانوي 100 نانوميتر والفضة النانوية على التوالي وكان هناك فرق معنوي ما بين المعاملتين كلتاهما. كما يبين الجدول رقم (7) أن نسبة فقس البيض تأثرت بالتراكيز المستعملة في هذه الدراسة بلغت أعلى

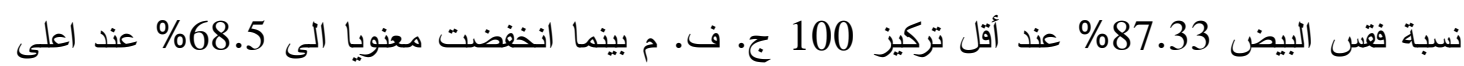
تركيز في الدراسة.

كما يبين التداخل ما بين نوع المادة النانوية وتركيزها أيضا التأثير المعنوي فبلغت اقل نسبة للفقس

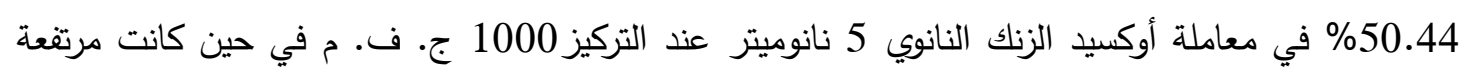

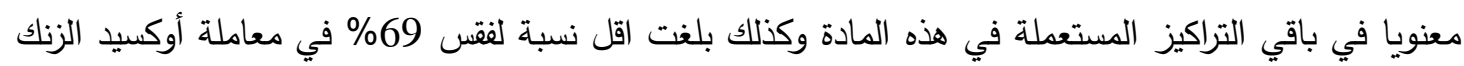

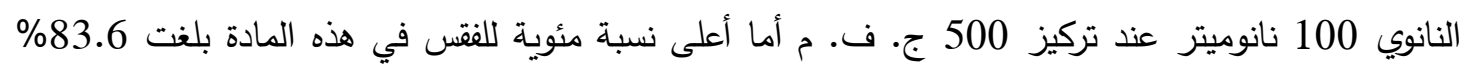
عند التركيز 1000 ج. ف. م وكان هناك فرق معنوي فيما بين المعاملة التي سبقتها.

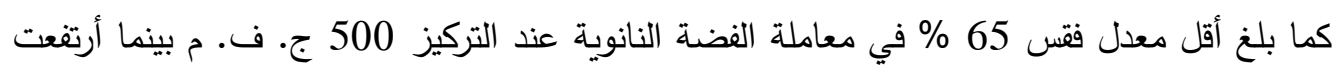

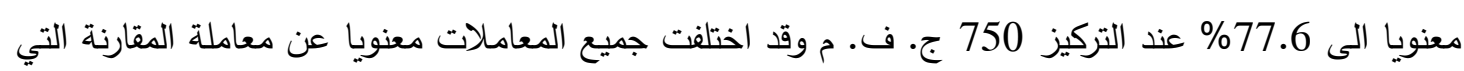
بلغت 98\% اي يمكن القول ان المعاملات اثرت معنويا في خفض حيوية البيوض الناتجة من الإناث المعاملة في سبق ان عوملت اطوارها بالمواد النانوية قيد الدراسة. يمكن القول أن المعاملة أعلاه خفضت نسبة الفقس الى 50\% بالى ولم نجد في في الدوريات دراسة مشابهة لمقارنة نتائجنا أعلاه .

الجدول رقم (7): تأثير تراكيز مختلفة لثلاث مواد نانوية على نسبة فقس البيض للجيل الاول لخنفساء اللوبيا الجنوبية Callosobruchus maculatus

\begin{tabular}{|c|c|c|c|c|c|c|}
\hline \multirow{2}{*}{ معدل تأثير المادة } & \multicolumn{5}{|c|}{ نسبة الفقس البيض للجيل الأول } & \multirow{2}{*}{ المادة } \\
\hline & 1000 & 750 & 500 & 250 & 100 & \\
\hline $\begin{array}{c}84.34 \\
\text { c }\end{array}$ & $\begin{array}{c}50.44 \\
\mathrm{i}\end{array}$ & $\begin{array}{c}90 \\
\mathrm{~B}\end{array}$ & $\begin{array}{c}91.3 \\
\text { b }\end{array}$ & $\begin{array}{c}97 \\
\mathrm{a}\end{array}$ & $\begin{array}{l}93 \\
a-b\end{array}$ & أوكسيد الزنك النانوي حجم (5) نانوميتر \\
\hline $\begin{array}{c}76.32 \\
\mathrm{~d}\end{array}$ & $\begin{array}{c}83.6 \\
\mathrm{c}\end{array}$ & $\begin{array}{l}76 \\
\text { e-f }\end{array}$ & $\begin{array}{l}69 \\
\text { g-h }\end{array}$ & $\begin{array}{l}82 \\
c-d\end{array}$ & $\begin{array}{l}71 \\
f-g\end{array}$ & أوكسيد الزنك النانوي حجم (100) نانوميتر \\
\hline $\begin{array}{c}71.3 \\
\mathrm{c}\end{array}$ & $\begin{array}{c}71.6 \\
\mathrm{f}-\mathrm{g}\end{array}$ & $\begin{array}{c}77.6 \\
\mathrm{E}\end{array}$ & $\begin{array}{c}65 \\
\mathrm{~h}\end{array}$ & $\begin{array}{l}75 \\
\text { e-f }\end{array}$ & $\begin{array}{c}67.3 \\
\text { g-h }\end{array}$ & الفضة النانوية حجم (100) نانوميتر \\
\hline \multicolumn{6}{|c|}{$\begin{array}{c}98 \\
\text { A }\end{array}$} & 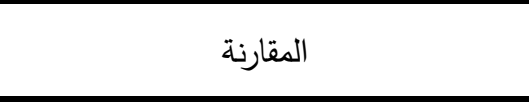 \\
\hline & $\begin{array}{c}68.5 \\
\mathrm{e}\end{array}$ & $\begin{array}{c}81.2 \\
\text { C }\end{array}$ & $\begin{array}{c}75.1 \\
\mathrm{~d}\end{array}$ & $\begin{array}{c}84.66 \\
\text { b }\end{array}$ & $\begin{array}{c}87.33 \\
\mathrm{a}\end{array}$ & معدل تأثير التركيز \\
\hline
\end{tabular}

* الارقام التي تحمل أحرفاً مختلفة في الحقل نفسة تختلف عن بعضها احصائيا حسب اختبار دنكن المتعدد المدى وعند مستوى احتمال5\%.

المصادر 
1) Mehdi, Mohamed Taher and Radi Hammadi (1987). Effect of the use of some powders and protective oils on the biology of the southern cowpea beetle. Callosobruchus maculatu (Coleopteran: Bruchidae) Arab Plant Protection Journal 5: 48-52.

2) Al bakr ,Azhar Abdul-Jabbar Hamid (1999). Effect of the plant Melia azedarach $\mathrm{L}$ in the life of the Southern cowpea beetle Callosobruchus maculatus. Master Thesis. College of Education, University of Mosul.

3) Ismail, Ayad Yousef Al-Haj (2005). Internet Portal to Entomology Sites, College of Education, University of Mosul.

4) Al-Azzawi, Abdullah Falih, Ibrahim Kadouri Qado Wahid Saleh alJundari (1990) Economic insects, Dar al-Hikma for printing and publishing, University of Baghdad, p. 652..

5) Athanassiou, C. G; Kavallieratos N. G; Benelli, G.; Losic and D. P. Usha Rani Desneux. N (2017). (Nanoparticles for pest control: current status and future) perspectives Volume 91Issue1 pp 1-15.

6) Barik TK; Sahu, B. and Swain, V. (2008). Nanosilica-from medicine to pest control. Parasitol Res 103:253-258.

7) Wang, X.; Ding, Y.; Summer, C. j., and Wang, (2004). Large-scale Synthesis of six nanometer-wide $\mathrm{ZnO}$ nanobelts "journal of physical Chemistry $\mathrm{B}$, vol. 108 $10-26$.

8) Lee, K. J; Nallathamby, P.D.; Browning, L.M.; Osgood, C.J. and Xu, XHN (2007). In Vivo imaging of transport and biocompatibility of Single silver nanoparticles in early development of Zebrafish embryos ACS, Nanos 1(2):133_143.

9) Owolade, O. F.; Ogunleti, D. O. and Adenekan, M. O. (2008). Titanium Dioxide affects disease development and yield of edible cowpea. Electronic Journal of Environmental, Agricultural and Food Chemistry, 7(50): 2942-2947.

10) Szentest, A. (1972). Studies on the mass rearing of Acanthoscellides obtecus Say (Coleoptera: Bruchide). Acta Phytopathologia Acadmic Scientiarum Hungaricas, 7(4), 453-463.

11) Ma, D.; Zalucki M. and Gordh ,G. (2002).aComparison of abundance of Helliverpa ssp. and predators between Conventional and Bt cotton .Report to Rhone - poulence Rural Australia PTX Ltd ,.Report - II ,25- 38.

12) Al-Rawi, Khasha Mahmoud and Khalafallah, Abdul Aziz Mohammed (2000). Design and analysis of agricultural experiments. Ministry of Higher Education and Scientific Research, Publishing House for Printing and Publishing, University of Mosul ,488..

13) Rouhani, M.; Samih, M.A. and Kalantri, S. (2012). Insecticidal effect of silica and silver nanoparticles on the cowpea seed beetle, Callosobruchus maculatus F. (Col.: Bruchidae).J. Entomol Res 4:297-305.

14) Malaikozhunda, B.; and Vinodhinib, J. (2018). Nanopesticidal effects of Pongamia pinnata leaf extract coated zinc oxide nanoparticle against the Pulse beetle Callosobruchus maculatus F. https://doi.org/10.1016/j.mtcomm.2017.12.015.

15) Rouhani, M. M. A. Samih and S. Kalantari, (2013). Insecticide effect of silver and zinc nanoparticles against Aphis nerii Boyer De Fonscolombe (Hemiptera: Aphididae). Chilean J. Agric. Res., 72: 590- 594.

16) Bendari, Hilmi Mohammed. Abeer Salah Eddine and Alexander Majid Lina Ahmed Helali (2013). Determination of Neo-toxicity of Nano Oxide and Nano Zinc Oxide against Sousse Rice as a promising insecticide, Egyptian Journal of Zoology.

17) Sahayaraj, K.; Madasamy, M. and Anbu, Radhika, S. (2016). Insecticidal activity of bio-silver and gold nanoparticles against Pericallia ricini Fab. (Lepidaptera: Archidae) JBiopest 5(1): 1-6 (c) 494-59. 